\title{
Evaluation of a Technique to Simplify Depictions of Visually Complex Aeronautical Procedures for NextGen
}

\author{
Divya C. Chandra and Rebecca Grayhem \\ United States Department of Transportation Volpe National Transportation Systems Center \\ Cambridge, Massachusetts
}

\begin{abstract}
Performance based navigation supports the design of more precise flight procedures. However, these new procedures can be visually complex, which may impact the usability of charts that depict the procedures. The purpose of the study was to evaluate whether there are performance benefits from simplifying aeronautical charts that depict visually complex flight procedures by separating the procedures onto different chart images. Forty-seven professional pilots participated. They used high-fidelity current and modified charts to find specific information from approach and Standard Instrument Departure (SID) chart images that were shown one at a time on a computer monitor. Response time and accuracy were recorded. Results show a consistent and significant reduction in the time to find information from the simplified chart images. Response time varied linearly with a simple clutter metric, the sum of visual elements in the depiction, indicating serial visual search. Most questions were answered with high accuracy, but some questions about altitude constraints yielded low accuracies.
\end{abstract}

\section{INTRODUCTION}

The Federal Aviation Administration (FAA) and International Civil Aviation Organization (ICAO) are transitioning to performance based navigation $(\mathrm{PBN})$ operations. PBN operations are a key component of the evolution of the National Airspace System (NAS) towards the Next Generation Air Transportation System (NextGen).

Aeronautical procedures designed with PBN technologies such as area navigation (RNAV) and required navigation performance (RNP) offer safety enhancements along with new levels of flexibility to negotiate terrain, airspace, and environmental considerations. More RNAV procedures, with and without RNP segments, are being developed each year to support PBN (FAA, 2012).

PBN procedures bring challenges for human performance because the flight paths must be flown more precisely. There are more altitude and speed constraints for the pilot to manage and more notes for the pilot to interpret. Pilots are specially trained to perform these procedures with the aid of various levels and types of flight deck automation. They must be able to understand the flight path, determine equipage requirements, understand RNAV and RNP terminology used by Air Traffic Control, and understand how to interpret flight deck automation and alerting interfaces properly for these procedures (FAA 2007, 2009, and 2011).

One consequence of new $\mathrm{PBN}$ procedures is that depictions of the procedures on aeronautical charts must show more data to describe the path fully. Chandra, Grayhem, and Butchibabu (2012) describe the various charting challenges and strategies that are used to handle them.

For example, one benefit of RNAV and RNP is that there can be more unique paths in a single procedure. This has the effect that the charted depictions differ from one another more than they do for conventional procedures, such as for an Instrument Landing System (ILS). This situation is illustrated in Figures 1 and 2. Figure 1 shows the plan view of a conventional ILS approach into Boise, Idaho. An RNAV (RNP) approach in to the same runway is shown in Figure 2. There are more flight path transitions and more segments for each flight path (i.e., more turns and altitude changes) in the RNAV (RNP) procedure than in the conventional ILS approach procedure.

If the procedure design is fixed, there are only three ways to simplify the charted depiction (Chandra et al., 2012). One option is to use nonstandard graphical techniques, such as larger pages or off-scale insets. A second option is to separate

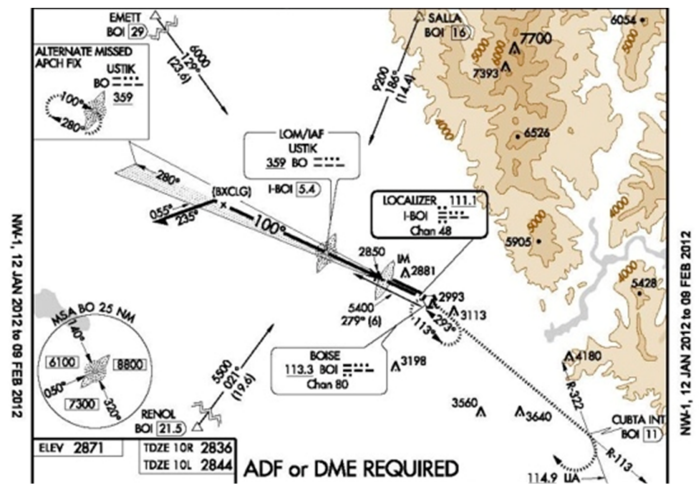

Figure 1. Plan view of Boise ILS approach.

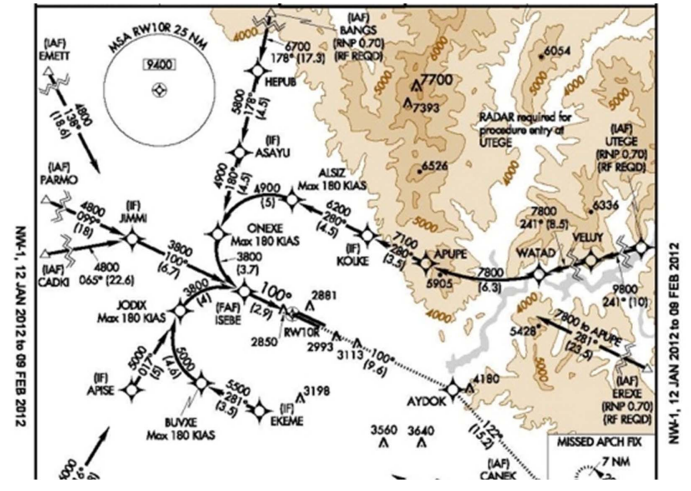

Figure 2. Plan view of Boise RNAV (RNP) approach. 
the procedure depiction onto different chart images, creating more pages. Finally, information for the procedure may be removed entirely. For example, data that are not pertinent to the specific operator using the procedure could be deleted.

In this study, we explored the effects of the second option, splitting information across more chart images. We expected that simplified charts with less information per image would yield faster search times for specific data. The study was designed and conducted jointly with the Massachusetts Institute of Technology (MIT). Preliminary results of the study were reported in Butchibabu, Grayhem, Hansman, and Chandra (2012). Since then we have analyzed the data further, yielding additional insights on how well the technique works. These updated analyses and results are the focus of this paper.

\section{METHOD}

The study description below is abbreviated. Additional information about the method, an extended discussion of results, and images of the stimuli are provided in a full government technical report (Chandra \& Grayhem, 2013). Chandra et al. (2012) provide background on the differences between approach and SID charts and procedures.

\section{Participants}

Data were collected from 47 RNP-qualified pilots (19 corporate and 28 airline) who operate in the United States. The corporate pilots had an average of 10,179 hours of flight experience and the airline pilots had an average of 12,056 flight hours. All of the participants used Jeppesen charts regularly. Some also had experience with FAA charts. All participants had received simulator training on RNAV procedures within the last 12 months. Most participants reported being comfortable flying RNAV SIDs. On a scale of 1 to 5,25 of the 47 pilots chose the highest comfort rating (5). Most pilots were also comfortable with RNAV (RNP) approaches; 34 of 48 rated comfort level as either a 4 or 5 .

\section{Procedure}

Each participant was first introduced to the study and signed an informed consent form. Participants then completed a background questionnaire to record their familiarity with RNAV and RNAV (RNP) procedures and information about their flight experience. Fourteen pilots were assigned to use FAA charts for the study and 33 used the Jeppesen charts. The pilots in the FAA-chart condition reviewed a short set of training slides that highlighted differences between the Jeppesen and FAA charting conventions.

Participants completed the task in two blocks with a rest period between blocks. One block was for approaches and the other block was for SIDs. The order of the approach and SID blocks was counterbalanced between participants. Chart modification (current or modified) was a within-subjects variable. Modified and current charts were presented in random order within the block.

The approach block contained six practice trials and 56 test trials, where each trial involved using a chart to answer one question. The SID block contained six practice trials and 44 test trials. Data from the practice trials were excluded from the analysis.

The experiment took approximately one hour to complete including instructions, breaks, and the questionnaire.

Participants in the FAA-chart condition spent 15 additional minutes on the differences training.

Stimuli. Current and modified Jeppesen and FAA charts were tested. Current charts show all paths on one chart image. Jeppesen and the FAA created the modified charts using their respective charting conventions. Specifications for the multiimage format were developed in coordination with Jeppesen and the FAA. Three main changes were made. First, we separated the procedure onto different chart images by erasing different flight paths from the current image. Figure 3 shows a current image with all the paths. Figure 4 shows one modified image created from the current chart in Figure 3. We did not rescale or re-center the modified chart images. Second, we added a line to the name of the modified chart image to indicate what transitions were shown on that image. Finally, we updated the profile view to correspond to the flight path.

Three visually complex approach procedures and three SIDs were tested. Each of the flight procedures was from a different airport. The RNAV (RNP) approaches were from Boise, Idaho (BOI), Bozeman, Montana (BZN), and Palm Springs, California (PSP). The RNAV SIDs were from Dallas-

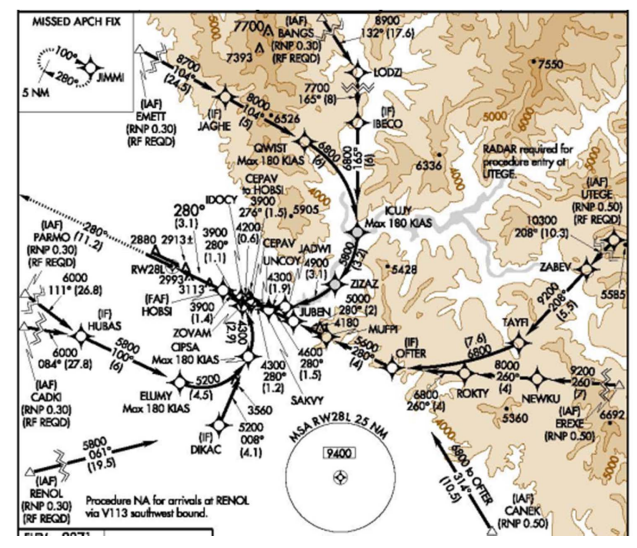

Figure 3. Plan view of current Boise chart image.

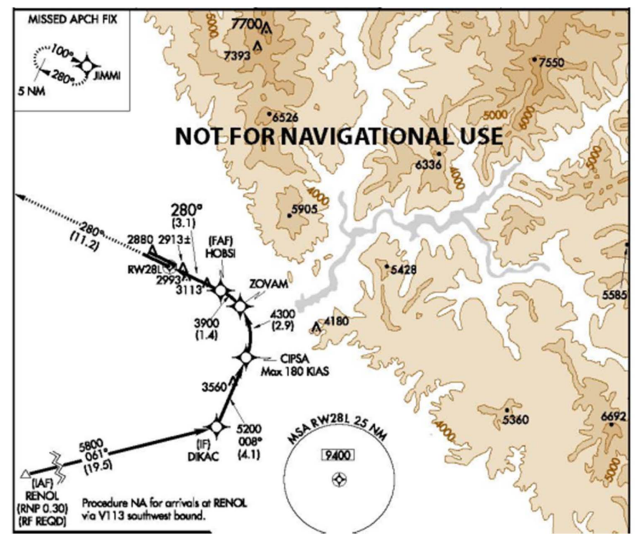

Figure 4. Plan view of Boise Renol chart image. 
Fort Worth, Texas (DFW), Las Vegas, Nevada (LAS), and Salt Lake City, Utah (SLC). The BOI approach was split into four chart images. The BZN, PSP, and SLC procedures were split into three images and the LAS and DFW procedures were split into two images. The number of modified chart images was related to the number of paths in the original chart. Arrival procedures were not selected for the study because these were generally less visually complex.

Task. For each trial in the study, participants first saw their expected route of flight and a question about that route. After they were oriented to the route, they called up the chart and searched for the answer. Their instructions were to answer the question as quickly and accurately as possible. The chart shown was always the correct one for the question and route; pilots did not have to search for the correct chart. The charts were displayed on a large desktop monitor (90 pixels/inch resolution). We recorded how much time the participant spent viewing the chart and his/her response.

We asked pilots to find information such as speeds, altitudes, distances, frequencies, and headings. There was always an equal number of questions for the current and modified charts, but the questions were never repeated so that participants could not memorize the answers.

\section{ANALYSES AND RESULTS}

\section{Response Times}

Response time was analyzed in different ways. First, we looked at the overall response times for current and modified charts separately for approaches and SIDs. Next, we examined how response times changed over the course of the hour-long experiment. Finally, we examined the relationship between the number of elements on the graphic portion of the chart (i.e., a rough estimate of "clutter") and the time required to find a specific piece of information from that chart.

Response time by chart modification and airport. Two repeated-measures analyses of variance were performed, one for approaches and the other for SIDs. The analyses were performed on the logarithm of response times (to normalize the data). Response times were first averaged across all of the modified chart images within a procedure for this analysis.

Figure 5 shows the mean response times for current and modified approaches and SIDs. The main effect of chart modification was highly significant. Pilots were just over 6 seconds faster when using modified approach charts $\left(F_{43}=\right.$ 261.38, $p<0.001)$ and almost 3 seconds faster with the modified SID charts $\left(F_{43}=56.68, p<0.001\right)$.

Figure 6 illustrates the main effect of airport, which was also significant $\left(F_{42}=44.17, \mathrm{p}<0.001\right.$ for approaches and $F_{42}$ $=5.96, \mathrm{p}=0.005$ for SIDs). Pairwise t-tests indicate that average times to find information for the Boise approach chart were significantly longer than for the other two approach charts. Response times for Salt Lake City were significantly longer than for Dallas Fort-Worth and Las Vegas.

Figure 6 also shows that the benefits of the chart modification were consistent across airports. Pairwise t-tests indicated that the differences between current and modified charts were statistically significant for every procedure (all $t_{46}$ greater than 3.4).

Response time interactions. A significant interaction between chart modification and airport can be seen in Figure 6 for approaches, indicating that different charts benefitted to different extents from the modifications $\left(F_{42}=26.00, \mathrm{p}<\right.$ 0.001). Response times were significantly faster for the BOI modified charts than for PSP and BZN for both FAA and Jeppesen charting conventions. For SIDs, a three-way interaction was discovered between chart modification, airport, and charting convention $\left(F_{42}=7.54, \mathrm{p}<0.01\right)$, indicating that some airports benefitted more from the chart modifications than others and these benefits were specific to the charting convention. For example, response times for the FAA SID chart for DFW improved the most with the chart modifications. These interactions are discussed further in Chandra and Grayhem (2013).

Response time by trial number. We were interested to know whether response times varied systematically over the course of the experiment. A systematic decrease in response times would indicate that participants learned the charts over time. A systematic increase in response times would indicate fatigue. To check for learning or fatigue, we correlated trial number with response time, because trial number is a record of when the trial occurred within the experiment.

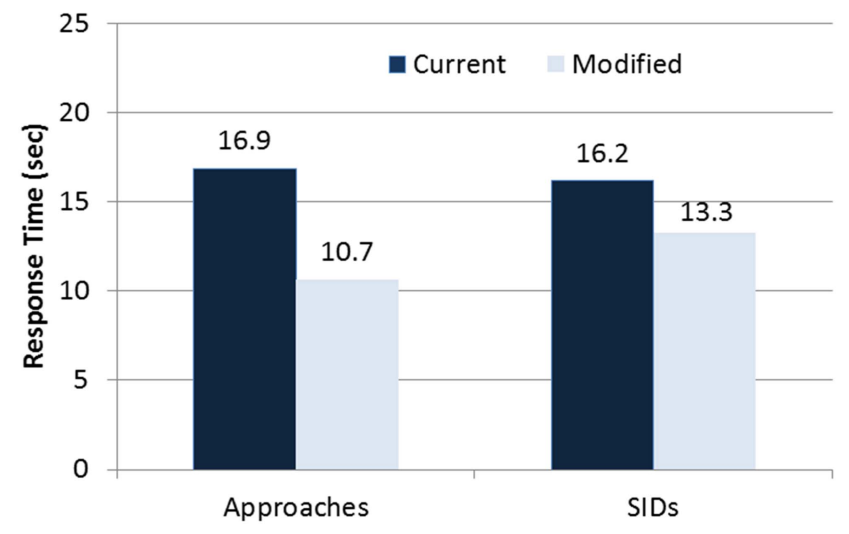

Figure 5. Mean response times for current and modified charts, by type of procedure.

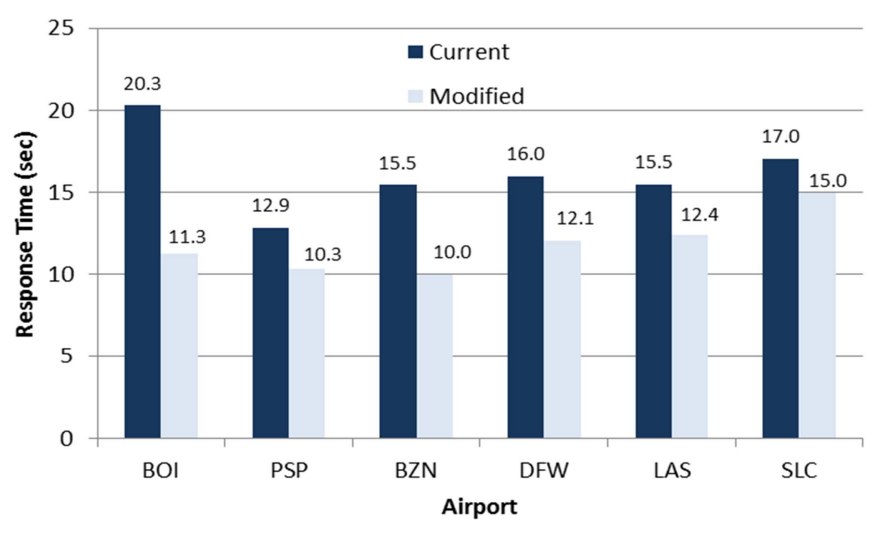

Figure 6. Mean response times for current and modified charts by airport. 
The test indicated a small effect in the direction of learning. Response times decreased over time for both SIDs $\left(t_{46}=9.37, p<0.001\right)$ and approaches $\left(t_{46}=9.40, p<0.001\right)$. Correlations for individual participants ranged from -0.39 to 0.16 with an average of -0.18 for SIDs and from -0.55 to 0.13 with an average of -0.19 for Approaches.

Response time by element count. The main difference between modified and current charts was that some paths were erased to create the modified charts. In essence, we removed some information from the chart plan view. We hypothesized that the improved performance with modified charts could be modeled if we were able to quantify how much information was removed. This hypothesis makes sense in the framework of a visual search task, where the desired piece of information is the "target" and all other elements are "distracters" (see Treisman \& Gelade, 1980, Wickens \& McCarley, 2008, and Wolfe, 1998). Often in visual search tasks, search is random and serial, as indicated by a response time to find the target that varies linearly with the number of distracters (except in specific situations where parallel processing is effective, e.g., when the target color is unique).

In order to quantify how much information was removed in the modified chart, we constructed a simple metric for how much information was on the chart: the number of elements in the graphic view of the chart (the main area where modifications were made). For approaches, we only counted elements in the plan view, and for SIDs we counted similar elements, but these could be found anywhere on the graphical view. The actual metric was based on a count of elements on the FAA versions of the chart. There may be some differences if the element count were based on the Jeppesen charts due to variations in the charting conventions. However, we expect these differences to be small because both manufacturers use the same source data to produce the charts, and because the differences of interest are likely to be relative (between current and modified charts), not absolute.

Table 1 lists the chart elements that we counted. Each element was given equal weight; the count was simply incremented by 1 for each element. A high element count indicates more data on the chart, while a low count indicates a simple chart page. Obstruction altitudes were not counted for the analysis because terrain elements (i.e., the shaded contours and peak altitudes) were not modified in the chart prototypes. As an example, the current Boise approach image (Figure 3) had 142 chart elements, whereas modifications resulting in only one path on the image, such as the BOI Renol image (Figure 4), had only 22 elements. Each modified chart image was scored for clutter individually.

We used a bivariate linear regression to compute the regression equation and correlation coefficient for element count and response time. First, however, we removed outliers, which were defined conservatively as response times greater than 60 seconds (1.8\% of approach trials and $1.8 \%$ of SID trials). We also removed one confusing question from the SID trials that had many excessive response times (1.6\%). With this clean set of data, results indicate a strong positive linear relationship between the number of elements and response times. For approaches, the correlation coefficient was 0.87 and for SIDs, 0.88 , both highly statistically significant. Across approaches and SIDs, the slope of the regression line was $66 \mathrm{msec}$ (per element) and the intercept was 8.5 seconds.

Table 1. Elements counted for clutter metric.

\begin{tabular}{lc}
\hline \multicolumn{1}{c}{$\begin{array}{c}\text { Elements Counted for Both } \\
\text { Approaches and SIDs }\end{array}$} & $\begin{array}{c}\text { Boise Approach Renol } \\
\text { Image Example Count }\end{array}$ \\
\hline Minimum En route Altitudes & 4 \\
Headings & 3 \\
Distances & 5 \\
Waypoints & 5 \\
Altitude Restrictions & 0 \\
Speed Restrictions & 1 \\
Notes $\quad$ Additional Elements Counted for Approaches \\
\hline \multicolumn{2}{c}{2} \\
\hline Radius-to-Fix Legs & 1 \\
Holding Patterns & 1 \\
\hline \multicolumn{2}{c}{ Additional Element Counted for SIDs } \\
\hline Minimum Obstruction Clearance Altitudes (MOCAs)
\end{tabular}

\section{Accuracy}

Pilot responses were scored manually for accuracy. The vast majority of questions, 84 out of 102, were answered with better than $90 \%$ accuracy. Of the 18 low-accuracy questions, 11 asked for altitude constraints, so we explored these questions further. Two questions were excluded because they referred to an outdated convention for depicting procedural altitudes. We also excluded data from five participants who did not indicate "above" or "below" for any of the altitude questions, which implied that they may have misunderstood the task.

There were still four questions with accuracies below $90 \%$ in the clean data. Two questions had accuracy rates of just $64 \%$ and $55 \%$. Both of these questions asked for an "at or below" altitude constraint on the Palm Springs approach chart.

\section{DISCUSSION}

The main finding of this study is that pilots are able to find information more quickly from chart images that have been simplified to show less information. This effect is strong and consistent across chart types, charting conventions, and airports tested. In the context of previous research on visual search, this is not a surprising result, although the strength of the result was not expected. We also found evidence that benefits of the modification technique are specific to the procedure, and even to the charting convention in some cases. This result is also intuitive. The modified charts used in the task are similar to charts that might be produced by a datadriven electronic chart, so we expect that the benefits of this technique would extend to electronic chart applications that can remove information from charts quickly and effectively.

Interestingly, we found evidence for a serial search strategy for this task, as indicated by the strong correlation between our simple clutter metric and response times. This result supports the idea that pilots are using bottom-up processing rather than top-down processing to find the information they need. Pilots may feel that they are able to filter out irrelevant flight paths quickly and effectively once 
they comprehend their route clearance, but there is still a measurable impact of showing irrelevant information.

Although the clutter metric was simple, it was effective in comparing plan views. If the visual search model is appropriate, and we believe it is, then the findings of this study could well be generalized to other types of aeronautical charts, beyond RNAV SIDs and RNAV (RNP) approaches. We do not expect that pilot training and operational experience related to RNP-qualification affected the results (other than that pilots understood the context and questions quickly) because visual search is a basic perceptual process.

One caveat on the implications of our response-time results is that they apply only to the type of task we gave pilots, which was to look for one specific piece of information using the chart. In practice, charts are also used for planning and orientation, which are not directed search tasks. Also, in practice, pilots could find some of the information we requested on flight deck displays without using a chart. Finally, we did not ask pilots to locate the correct chart, which is a task they would normally have to do. However, that task is usually performed in low time-pressure conditions (pre-flight) and therefore may not represent a comparable tradeoff.

We also found that some pilots misinterpreted altitude information in certain cases. There is little data on this issue, but enough to warrant a more focused study. We found that pilots misread some "at or below" constraints, which are uncommon on approach procedures where pilots usually stay "at or above" given altitudes, for terrain avoidance. Pilots may have misinterpreted the "at or below" altitudes on the approach because they violated routine expectations, because they misread the graphic depiction of the constraint, or because altitudes are inherently complex data.

\section{SUMMARY AND CONCLUSIONS}

We present a detailed analysis of data from a study to test the performance benefits of a specific chart-modification technique. The modifications were intended to reduce the time to find data from a chart by separating information from a complex chart onto multiple chart images. Our results showed that there are significant improvements in terms of time to find the information on the simplified charts. Response times can be modeled as a linear function of a simple chart clutter metric, the sum of visual elements on the graphic view.

This experiment represents a first step in understanding how to improve the usability of charts for visually complex procedures that are becoming more common in PBN operations. There are, however, many practical factors that were not addressed. For example, we made custom modifications to a few carefully selected procedures. If this strategy is adopted, a more comprehensive process will need to be developed for deciding when and what modifications to make, taking into account factors such as cost of production of multiple chart images and the difficulties of handling more chart images.

\section{ACKNOWLEDGMENTS}

This paper was prepared by the Aviation Human Factors Division of the Center for Human Factors Research and System Applications at the Volpe Center. The FAA NextGen Human Factors Division (ANG-C1) coordinated the research requirement, and its principal representatives (Dan Herschler and Tom McCloy) acquired, funded, and technically managed execution of the research services described in this report. We thank Kathy Abbott (AVS) and Mark Steinbicker (AFS-470) for their input on this effort. We would also like to thank all the subject matter experts and pilots who helped us.

The modified chart prototypes tested in this experiment were created by FAA Aeronautical Navigation Products and Jeppesen, Inc. Thanks to John Moore, Valerie Watson, and Alex Rushton from the FAA and Ted Thompson and Jeff Williams from Jeppesen.

Ms. Abhizna Butchibabu performed this study as part of her work towards a Master's degree under Professor R. John Hansman in the MIT Department of Aeronautics and Astronautics. MIT was funded by Volpe Center through Contract No. DTR57-07-D-30006. Thanks also to Andrew Kendra, Alan Midkiff (MIT), and Alan Yost for their assistance with the experiment, to Michael Zuschlag for his invaluable advice on the data analysis, and to Maura Lohrenz for feedback on the paper.

The views expressed herein are those of the authors and do not necessarily reflect the views of the Volpe National Transportation Systems Center, the Research and Innovative Technology Administration, or the United States Department of Transportation.

\section{REFERENCES}

Butchibabu, A. Grayhem, R.J., Hansman, R.J., \& Chandra, D.C. (2012) Evaluating a de-cluttering technique for NextGen RNAV and RNP charts. Proceedings of $31^{\text {st }}$ Digital Avionics Systems Conference (DASC), 14-18 October 2012, Williamsburg, VA. www.volpe.dot.gov

Chandra, D. C., Grayhem, R.J., \& Butchibabu, A. (2012). Area Navigation and Required Navigation Performance Procedures and Depictions (US DOT Volpe National Transportation Systems Center, DOT/FAA/TC-12/8 and DOT-VNTSC-FAA-12-10). Cambridge, MA. www.volpe.dot.gov

Chandra, D. C. \& Grayhem, R.J. (2013). Evaluation of a Technique to Simplify Area Navigation and Required Navigation Performance Charts (US DOT Volpe National Transportation Systems Center, DOT-VNTSCFAA-13-02). Cambridge, MA. www.volpe.dot.gov

Federal Aviation Administration. (2007). US Terminal and En Route Area Navigation Operations (Advisory Circular 90-100A). Washington, DC.: www.faa.gov

Federal Aviation Administration. (2009). Approval Guidance for RNP Operations and Barometric Vertical Navigation in the U.S. National Airspace System. (Advisory Circular 90-105), Washington, DC.: www.faa.gov

Federal Aviation Administration. (2011). Approval Guidance for RNP Procedures with Special Aircraft and Aircrew Authorization Required (AR) (Advisory Circular 90-101A). Washington, DC. www.faa.gov

Federal Aviation Administration. (2012). NextGen Implementation Plan 2012, www.faa.gov/nextgen/implementation/plan.

Treisman, A. M. \& Gelade G. (1980).A feature-integration theory of attention. Cognitive Psychology, 12(1), pp. 97-136.

Wickens, C. D. \& McCarley J.S. (2008). Applied attention theory. Boca Raton, FL: CRC Press Taylor \& Francis Group. 